\title{
openheart Right heart function during simulated altitude in patients with pulmonary arterial hypertension
}

\author{
Leigh M Seccombe, ${ }^{1,2}$ Vincent Chow, ${ }^{2,3}$ Wei Zhao, ${ }^{3}$ Edmund M T Lau, ${ }^{2}$ \\ Peter G Rogers, ${ }^{1}$ Austin C C Ng, ${ }^{2,3}$ Elizabeth M Veitch, ${ }^{1}$ Matthew J Peters, ${ }^{1,2}$ \\ Leonard Kritharides ${ }^{2,3}$
}

To cite: Seccombe LM, Chow V, Zhao W, et al. Right heart function during simulated altitude in patients with pulmonary arterial hypertension. Open Heart 2017:4:e000532.

doi:10.1136/openhrt-2016000532

Received 7 September 2016 Revised 21 November 2016 Accepted 22 November 2016

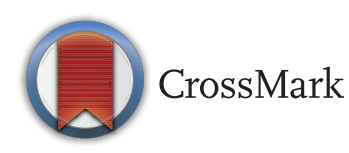

${ }^{1}$ Department of Thoracic Medicine, Concord Repatriation General Hospital, Concord, New South Wales, Australia

${ }^{2}$ Sydney Medical School, The University of Sydney, Sydney, Australia

${ }^{3}$ Department of Cardiology, Concord Repatriation General Hospital, Concord, New South Wales, Australia

\section{Correspondence to} Dr Leigh M Seccombe; leigh. seccombe@sswahs.nsw.gov. $\mathrm{au}$

\section{ABSTRACT}

Objective: Patients with pulmonary arterial hypertension (PAH) are often recommended supplemental oxygen for altitude travel due to the possible deleterious effects of hypoxia on pulmonary haemodynamics and right heart function. This includes commercial aircraft travel; however, the direct effects and potential risks are unknown.

Methods: Doppler echocardiography and gas exchange measures were investigated in group 1 patients with PAH and healthy patients at rest breathing room air and while breathing $15.1 \%$ oxygen, at rest for 20 min and during mild exertion.

Results: The 14 patients with PAH studied were clinically stable on PAH-specific therapy, with functional class II $(n=11)$ and III $(n=3)$ symptoms when tested. Measures of right ventricular size and function were significantly different in the PAH group at baseline as compared to 7 healthy patients $(p<0.04)$. There was no evidence of progressive right ventricular deterioration during hypoxia at rest or under exertion. Pulmonary arterial systolic pressure (PASP) increased in both groups during hypoxia $(p<0.01)$. PASP in hypoxia correlated strongly with baseline PASP $(p<0.01)$. Pressure of arterial oxygen correlated with PASP in hypoxia $(p<0.03)$ but not at baseline, with three patients with PAH experiencing significant desaturation. The duration and extent of hypoxia in this study was tolerated well despite a mild increase in symptoms of breathlessness $(p<0.01)$.

Conclusions: Non-invasive measures of right heart function in group 1 patients with PAH on vasodilator treatment demonstrated a predictable rise in PASP during short-term simulated hypoxia that was not associated with a deterioration in right heart function.

\section{INTRODUCTION}

The clinical question of whether patients with cardiopulmonary disease can travel safely to higher altitude destinations is arising more frequently. There is an increased ease and affordability of air, train and road transport and an increasing number of travellers will have chronic disease. The emergence of

\section{KEY QUESTIONS}

What is already known about this subject?

- Patients with pulmonary arterial hypertension may experience deleterious effects of exposure to altitude-related hypoxia due to further elevated pulmonary artery pressure from increased sympathetic activity and hypoxic pulmonary vasoconstriction. The effects of altitude, comparable to commercial aircraft flight, on right ventricle function in these patients are unknown.

What does this study add?

- This novel study investigated the effects of mild altitude simulation on right ventricular function in patients with pulmonary arterial hypertension. Pulmonary artery pressure increased significantly during hypoxia, with no evidence of progressive right ventricular deterioration at rest or under mild exertion.

How might this impact on clinical practice?

- The clinical question of whether patients with pulmonary arterial hypertension can travel safely to higher altitude is arising more frequently and current recommendations are based on low levels of evidence. This primary study demonstrates that these patients can tolerate shortterm hypoxia well and further investigation can be safely considered.

targeted therapy has improved outcomes and quality of life in patients with pulmonary arterial hypertension (PAH). ${ }^{1}$ Owing to the possible negative physiological effects of hypoxia, it is recommended that those with the WHO functional class (FC) symptoms III and IV should not be exposed to 1500$2000 \mathrm{~m}$ altitude and above without supplemental oxygen. ${ }^{2}{ }^{3}$ This includes commercial aircraft travel where, although variable, the minimum pressure inside the cabin is $574 \mathrm{~mm} \mathrm{Hg}$, equivalent to $2400 \mathrm{~m}$ altitude, or breathing $15.1 \%$ oxygen at sea level. ${ }^{45}$ While the limited available evidence has reported 
minimal symptoms and arterial desaturation of $5-10 \%$ in flight and during simulation studies in patients with FC I-IV PAH, ${ }^{6}{ }^{7}$ there is no information regarding right ventricular (RV) function at altitude that may provide more appropriate insight into potential hypoxic stress and risk of a medically adverse event.

Arterial desaturation from hypoxic exposure is mitigated by increased sympathetic activity that elevates cardiac output. ${ }^{8}$ Increased pulmonary vascular resistance has been shown to occur in the presence of hypoxic pulmonary vasoconstriction that can occur within minutes of exposure to altitudes from 1500 to $2400 \mathrm{~m} .{ }^{9}{ }^{10}$ These combined effects result in higher pulmonary artery pressures at altitude than observed at the comparative cardiac output at sea level ${ }^{11}$ with increased afterload that may induce changes in RV function. ${ }^{12}$ For those with $\mathrm{PAH}$, any further elevation in pulmonary artery pressures may further impair RV function that subsequently may compromise the ability to adequately elevate cardiac output to combat hypoxia, particularly during exertion. However, no previous studies have evaluated the effects of hypoxia (at a level comparable to commercial air travel) on RV function in patients with PAH.

We sought to describe the effects of simulated $2400 \mathrm{~m}$ altitude at rest and during a mild exercise task on measures of RV function and gas exchange in patients with $\mathrm{PAH}$ and in a comparison group of healthy normal patients.

\section{METHODS}

\section{Subjects}

Patients with known PAH were recruited from a University Hospital Pulmonary Hypertension clinic. PAH was diagnosed in accordance with the European Society of Cardiology ${ }^{2}$ and American Heart Association guidelines. ${ }^{13}$ Patients with PAH included in the present study fulfilled both the following criteria: (1) Confirmed PAH (group 1) which had been initially diagnosed on right heart catheterisation ${ }^{2}{ }^{13}$ and (2) clinically stable patients (WHO FC symptoms III or less, with no hospitalisation for PAH deterioration in the past 12 months) on pulmonary vasodilator therapy. Exclusion criteria included uncontrolled hypertension, those requiring supplemental oxygen at sea level, neurological disease, structural abnormalities of the chest wall, recent upper respiratory tract infection or claustrophobia. Healthy normal patients without known heart or lung disease were recruited as a comparison group. All testing was performed with the patient taking their usual medications, including their pulmonary hypertension therapy. Medical history and current medications were sourced and documented from the patient's treating physician.

\section{Lung function}

Spirometry and diffusing capacity were performed according to the American Thoracic Society/European
Respiratory Society criteria (MasterScreen Body, Jaeger, Hoechberg, Germany). ${ }^{14}{ }^{15}$ Predicted values were derived from the recommendations of the Global Lung Initiative for spirometry and European Community for Coal and Steel for diffusing capacity. ${ }^{16} 17$

\section{Echocardiography}

All images were acquired from standard echocardiographic views in accordance with the American Society of Echocardiography recommendations. ${ }^{2}{ }^{18}$ All images were stored digitally and analysed offline using commercially available software (GE EchoPac 7.0.1, Horten, Norway). All prospectively acquired images were performed by an experienced operator (WZ) based on predetermined protocols. All analyses were performed in batches on anonymised images by a single analyst (VC) blinded to clinical details of patients. Resting echocardiography was performed to assess RV function and estimate the pulmonary artery systolic pressure (PASP). RV dimensions were measured at end-diastole from an RV-focused apical four-chamber view with care to avoid foreshortening and to ensure that the crux and apex of the heart were in view. The basal diameter is generally defined as the maximal short-axis dimension in the basal one-third of the RV seen on the four-chamber view. $^{19}$

The PASP was derived as the sum of the tricuspid regurgitant pressure gradient obtained from continuouswave Doppler and the right atrial pressure as estimated from the inferior vena cava assessment. ${ }^{2} \mathrm{RV}$ longitudinal systolic function was measured by determining the tricuspid annular plane systolic excursion (TAPSE) $^{2}$ and a cut-off of $<1.6 \mathrm{~cm}$ was considered abnormal in this study. ${ }^{20}$ The right-sided and left-sided cardiac chambers were analysed by conventional two-dimensional parameters that included: the ratio of RV and left ventricular (LV) end-diastolic diameters and the RV fractional area change (FAC), ${ }^{18}$ with an abnormal RV FAC being $<35 \%$. Global RV function was assessed using the modified Tei index, a validated measure of global ventricular function incorporating both systolic and diastolic functions based on tissue-Doppler imaging ${ }^{21}$ with an abnormal value defined as $>0.55 .{ }^{22}$ The $\mathrm{LV}$ ejection fraction was measured by the volumetric (Simpson's biplane) method, consistent with published guidelines. ${ }^{18} \mathrm{RV}$ dilation was defined as RV:LV end-diastolic area ratio $>0.6 .^{18}$ All measurements were averaged over three consecutive cardiac cycles. Intraobserver variability for individual echocardiographic parameters in our laboratory has been previously published ${ }^{23}$ and was derived from 20 patients, repeating all analyses on three separate occasions with the operator (VC) blinded to previous results.

\section{Hypoxic challenge}

While lying supine in a semirecumbent position, breath-by-breath gas analysis was monitored continuously (Oxycon Pro, Jaeger-Toennies, Hochberg, Germany) with the patient breathing through a large two-way, non- 
rebreathing valve (Y-shape 2730, Hans Rudolph, Kansas City, Missouri, USA) from a sealed face mask. Leaks were monitored and addressed when required to ensure that the fraction of inspired carbon dioxide was near $0 \%$ and fraction of inspired oxygen was stable. The system was calibrated for flow, gas concentration and response time prior to each test incorporating the current ambient conditions.

Measurements were initially collected at rest, while the subject breathed room air at sea level for at least $5 \mathrm{~min}$ ("baseline sea level"). Secondly subjects breathed hypoxic gas mix $\left(15.1 \%( \pm 0.2 \%) \mathrm{O}_{2}\right.$, balance $\mathrm{N}_{2}$ (BOC Gases, Australia)) from a $30 \mathrm{~L}$ gasbag reservoir for at least $20 \mathrm{~min}$ at rest ("hypoxia at rest"). This was immediately followed by at least 5 min of mild exercise while continuing to breathe the hypoxic gas mix ("hypoxia during exercise"). A radial arterial blood gas was only taken at 'baseline sea level' and 'hypoxia at rest' in patients with $\mathrm{PAH}$ and not during the exercise task. Mild exercise was in the form of leg raises while still semirecumbent, controlled to elicit a greater than twofold increase in $\mathrm{O}_{2}$ uptake (monitored continuously) from the patient's basal, resting metabolic rate. This is estimated to be $\approx 2$ Mets $(\approx 7-8 \mathrm{~mL} / \mathrm{min} / \mathrm{kg})$, reflective of the exertion from $<3 \mathrm{~km} /$ hours, level ground walking. ${ }^{24}$ Once the target $\mathrm{O}_{2}$ uptake was achieved, echocardiography was performed while the patient continued to leg-raise.

ECG and pulse oximetry were monitored continuously. Manual systemic blood pressure and symptoms of perceived breathlessness (modified BORG scale $0-10)^{25}$ were collected every $5 \mathrm{~min}$ and during mild exercise. Data are presented as mean (SD) unless otherwise stated. Ventilatory/gas exchange parameters are expressed as mean values derived from the final $5 \mathrm{~min}$ at 'baseline sea level' and 'hypoxia at rest' and the last minute in 'hypoxia during exercise'. The change in echocardiograph and ventilatory parameters between each phase were assessed using a two-way analysis of variance (ANOVA) rather than repeated measures as three patients with PAH did not complete all three test phases. Group comparisons were via an unpaired two-tailed t-test. Associations between measured variables were assessed using Pearson's correlation analysis. A p value $<0.05$ was considered statistically significant.

The study was reviewed and approved by the Human Ethics Review Board of Sydney Local Area Health District (New South Wales, Australia). Each patient gave written informed consent.

\section{RESULTS}

\section{Subjects and lung function}

Fourteen patients with PAH and seven healthy normal patients were recruited. Patient demographics and lung function are presented in table 1 .

All patients with PAH were in group 1 classification with the WHO FC III symptoms at diagnosis. Time since diagnosis and onset of treatment to study enrolment was 2.2 (1.8) years; all patients were clinically stable on PAH-specific therapy and presented with FC II $(n=11)$ and III $(n=3)$ symptoms when tested. Seven patients had idiopathic PAH and seven had PAH secondary to connective tissue disease. Four patients had hypertension, and two had diabetes mellitus. Seven patients with PAH were receiving an endothelin receptor antagonist (ERA), three were receiving a phosphodiesterase type 5 inhibitor (PDE5-I), and four were receiving combination therapy (ERA and PDE5-I). No study patients were receiving prostacyclin analogues, calcium channel blocker or $\beta$-blocker medications. There was no evidence

Table 1 Patient demographics, baseline lung function and initial diagnostic values

\begin{tabular}{|c|c|c|c|}
\hline & PAH & Control & p Value \\
\hline Male:female & $6: 8$ & $4: 3$ & - \\
\hline Age, years & $61(15)$ & $51(15)$ & 0.17 \\
\hline Height, $\mathrm{cm}$ & $167(12)$ & $166(9)$ & 0.78 \\
\hline BMI & $27.7(8.1)$ & $25.5(3.5)$ & 0.51 \\
\hline $\mathrm{FEV}_{1}, \mathrm{~L}$ & $2.18(0.98)$ & $3.36(0.84)$ & 0.02 \\
\hline Per cent predicted & $77(16)$ & $109(12)$ & \\
\hline FVC, L & $2.93(1.23)$ & $4.34(0.96)$ & 0.03 \\
\hline Per cent predicted & $81(15)$ & $112(10)$ & \\
\hline $\mathrm{K}_{\mathrm{co}}, \mathrm{mL} \cdot \mathrm{mm}$ Hg.min.L & $3.3(1.0)$ & $4.5(0.9)$ & 0.04 \\
\hline Per cent predicted & $59(17)$ & $92(10)$ & \\
\hline $\mathrm{V}_{\mathrm{A}}, \mathrm{L}$ & $4.53(1.45)$ & $5.54(0.72)$ & 0.01 \\
\hline Per cent predicted & 79 (12) & $107(14)$ & \\
\hline Mean $\mathrm{P}_{\mathrm{PA}}, \mathrm{mm} \mathrm{Hg}$ & $37(10)$ & - & \\
\hline PVR, woods units & $4.9(3.5)$ & - & \\
\hline 6MWT, m & $391(153)$ & - & \\
\hline Per cent predicted & $74(21)$ & - & \\
\hline
\end{tabular}

Data presented as mean (SD).

6MWT, 6 min walk test; BMI, body mass index; $F_{E V}$, forced expiratory volume in $1 \mathrm{~s}$; FVC, forced vital capacity; $\mathrm{K}_{\mathrm{CO}}$, transfer factor for carbon monoxide corrected for $\mathrm{V}_{\mathrm{A}}$; $\mathrm{PAH}$, pulmonary arterial hypertension; $\mathrm{P}_{\mathrm{PA}}$, pulmonary arterial pressure; PVR, pulmonary vascular resistance; $\mathrm{V}_{\mathrm{A}}$, alveolar volume. 
of significant LV dysfunction in any of the study patients. Forced vital capacity and alveolar volume were lower in the patients with PAH compared to controls $(\mathrm{p}<0.04)$. Diffusion capacity corrected for alveolar volume was reduced in the patients with PAH $(\mathrm{p}<0.05)$.

\section{Baseline gas exchange and echocardiographic parameters}

Baseline oxygen saturation was similar between the patients with PAH and controls $(97$ (1)\% and 98 (1)\%, respectively, $\mathrm{p}=0.21$ ), but the $\mathrm{PAH}$ group displayed a significantly higher respiratory rate and minute ventilation compared with controls $(\mathrm{p}<0.04)$. Overall, patients with PAH had moderately elevated PASP (56 (14) $\mathrm{mm} \mathrm{Hg}$ ) with RV dilation (RV:LV end diastolic area ratio 0.86 (0.39)). Measures of global and longitudinal RV size and function (modified Tei index, TAPSE, RV: LV diameter ratios and RV FAC\%) were all significantly different between the patients with PAH as compared to the control group $(\mathrm{p}<0.04)$. In the PAH group, $5 / 14(36 \%)$ had TAPSE $<1.6 \mathrm{~cm}, 9 / 14(64 \%)$ had Tei index $>0.55$ and $11 / 14(79 \%)$ had RV FAC $<35 \%$. Overall, 13/14 (93\%) of patients with PAH had at least one abnormal index of RV function.

\section{Hypoxia at rest and during exercise}

Echocardiographic images were obtained during hypoxia at rest in all patients. Three patients with $\mathrm{PAH}$ did not complete the exercise task and were not included in the 'hypoxia during exercise' analysis, two due to musculoskeletal issues and body habitus and one due to significant desaturation during resting hypoxia (pulse oximetry $<80 \%$ ). Three patients with $\mathrm{PAH}$ requested not to have an arterial blood gas during 'hypoxia at rest'. Serial echocardiograph and gas exchange parameters for each stage are presented in tables 2 and 3, respectively.

During hypoxia at rest and hypoxia during exertion, patients with $\mathrm{PAH}$ displayed lower oxygen saturation compared with controls $(\mathrm{p}<0.04$, table 3$)$. PASP at sea level correlated strongly with PASP during hypoxia, both at rest and during exercise (correlation coefficient 0.93, 0.89 , respectively, $\mathrm{p}<0.001)$. There was a correlation during 'hypoxia at rest' between pressure of arterial oxygen and PASP $(\mathrm{R}=-0.68, \mathrm{p}<0.03$, figure 1$)$ that was not evident at baseline in the patients with PAH $(p=0.2)$. Three patients with PAH experienced a significantly reduced pressure of arterial oxygen below $50 \mathrm{~mm} \mathrm{Hg}$ at rest during hypoxia with significantly elevated PASP that correlated with increased perception of breathlessness $(\mathrm{R}=-0.71, \mathrm{p}<0.03)$.

Within the PAH group, PASP increased from 56 (14) at baseline to 75 (17) $\mathrm{mm} \mathrm{Hg}$ during hypoxia with exertion $(p<0.01$, figure 2$)$. This increase in PASP was not accompanied by any significant changes in any right

\begin{tabular}{|c|c|c|c|c|}
\hline & Baseline sea level & Hypoxia at rest & Hypoxia during exercise $^{*}$ & p Value \\
\hline \multicolumn{5}{|c|}{ RV end-diastolic diameter } \\
\hline PAH & $41(7) \dagger$ & $40(8) \dagger$ & $40(8) \dagger$ & 0.87 \\
\hline Control & $29(8)$ & $22(9)$ & $29(8)$ & 0.20 \\
\hline \multicolumn{5}{|c|}{$\mathrm{RV}$ end-systolic area, $\mathrm{cm}^{2}$} \\
\hline PAH & $14(6) \dagger$ & $14(7) \dagger$ & $14(8) \dagger$ & 0.97 \\
\hline Control & $8(3)$ & 7 (2) & $6(2)$ & 0.21 \\
\hline \multicolumn{5}{|c|}{ RV:LV end-diastolic diameter ratio } \\
\hline $\mathrm{PAH}$ & $1.01(0.17) \dagger$ & $1.05(0.21) \dagger$ & $1.04(0.25) \dagger$ & 0.85 \\
\hline Control & $0.66(0.14)$ & $0.64(0.09)$ & $0.68(0.14)$ & 0.79 \\
\hline \multicolumn{5}{|c|}{ RV:LV end-diastolic area ratio } \\
\hline PAH & $0.86(0.39) \dagger$ & $0.99(0.40) \dagger$ & $1.04(0.40) \dagger$ & 0.47 \\
\hline Control & $0.43(0.08)$ & $0.61(0.15)$ & $0.51(0.11)$ & 0.03 \\
\hline \multicolumn{5}{|c|}{$\mathrm{RA}$ end-systolic area, $\mathrm{cm}^{2}$} \\
\hline $\mathrm{PAH}$ & $18(7)$ & $19(8) \dagger$ & $17(7) \dagger$ & 0.76 \\
\hline Control & $12(2)$ & $12(2)$ & $11(3)$ & 0.67 \\
\hline \multicolumn{5}{|l|}{ LV EF \% } \\
\hline PAH & $62(12)$ & $62(11)$ & $68(9)$ & 0.25 \\
\hline Control & $65(7)$ & $68(7)$ & $76(7)$ & 0.03 \\
\hline \multicolumn{5}{|l|}{$\mathrm{HR}, \mathrm{bpm}$} \\
\hline PAH & $76(13) \dagger$ & $78(12) \dagger$ & $94(16)$ & 0.02 \\
\hline Control & $62(7)$ & $65(8)$ & $88(9)$ & 0.01 \\
\hline \multicolumn{5}{|c|}{$\mathrm{SBP}, \mathrm{mm} \mathrm{Hg}$} \\
\hline PAH $\ddagger$ & $112(11)$ & $117(14)$ & $123(18)$ & 0.33 \\
\hline Control & $117(15)$ & $124(20)$ & $131(20)$ & 0.66 \\
\hline
\end{tabular}


Table 3 Gas exchange parameters

\begin{tabular}{|c|c|c|c|c|}
\hline & Baseline sea level & Hypoxia at rest & Hypoxia during exercise ${ }^{*}$ & p Value \\
\hline \multicolumn{5}{|l|}{$\mathrm{SpO}_{2}, \%$} \\
\hline PAH & 97 (1) & $90(4) \dagger$ & $91(2) \dagger$ & 0.01 \\
\hline Control & $98(1)$ & $94(2)$ & $94(2)$ & 0.01 \\
\hline \multicolumn{5}{|c|}{$\mathrm{PaO}_{2}, \mathrm{~mm} \mathrm{Hg}$} \\
\hline PAH & $86(10)$ & $55(6)^{*}$ & - & 0.01 \\
\hline \multicolumn{5}{|c|}{$\mathrm{PaCO}_{2}, \mathrm{~mm} \mathrm{Hg}$} \\
\hline PAH & $33(4)$ & $33(5)^{*}$ & - & 0.87 \\
\hline \multicolumn{5}{|l|}{$\mathrm{V}_{\mathrm{E}}, \mathrm{L} / \mathrm{min}$} \\
\hline PAH & $13.5(3.8) \dagger$ & $13.6(4.8) \dagger$ & $21.9(6.6)$ & 0.01 \\
\hline Control & $9.5(2.9)$ & $8.4(2.2)$ & $18.4(3.5)$ & 0.01 \\
\hline \multicolumn{5}{|l|}{$\mathrm{V}_{\mathrm{T}}, \mathrm{L}$} \\
\hline PAH & $0.73(0.25)$ & $0.71(0.24)$ & $1.11(0.45)$ & 0.01 \\
\hline Control & $0.67(0.10)$ & $0.66(0.13)$ & $0.97(0.23)$ & 0.01 \\
\hline \multicolumn{5}{|l|}{$\mathrm{RR}, \mathrm{bpm}$} \\
\hline PAH & $19(4) \dagger$ & $19(5) \dagger$ & $22(7)$ & 0.55 \\
\hline Control & $14(4)$ & $13(5)$ & $20(3)$ & 0.02 \\
\hline \multicolumn{5}{|c|}{$\mathrm{VO}_{2}, \mathrm{~mL} \cdot \min \cdot \mathrm{kg}$} \\
\hline $\mathrm{PAH}$ & $4.68(0.75)$ & $4.54(1.33)$ & $6.92(1.97)$ & 0.01 \\
\hline Control & $4.26(0.74)$ & $3.57(0.51)$ & $8.54(2.11)$ & 0.01 \\
\hline \multicolumn{5}{|l|}{ Borg $1-10$} \\
\hline PAH & $0.4(0.5) \dagger$ & $1.0(1.1) \dagger$ & $1.7(0.8) \dagger$ & 0.01 \\
\hline Control & $0.0(0.0)$ & $0.0(0.0)$ & $0.1(0.4)$ & 0.39 \\
\hline
\end{tabular}

Data presented as mean (SD). $\mathrm{N}=14 \mathrm{PAH}, \mathrm{N}=7$ Control.

${ }^{*} \mathrm{~N}=11 \mathrm{PAH}$.

tversus Control unpaired t-test $\mathrm{p}<0.05$.

$\mathrm{PAH}$, pulmonary arterial hypertension; $\mathrm{PaO}_{2}$, pressure of arterial oxygen; $\mathrm{RR}$, respiratory rate; $\mathrm{SpO}_{2}$, pulse oximetry; $\mathrm{V}_{\mathrm{E}}$, minute ventilation; $\mathrm{VO}_{2}$, oxygen uptake; $\mathrm{V}_{\mathrm{T}}$, tidal volume.

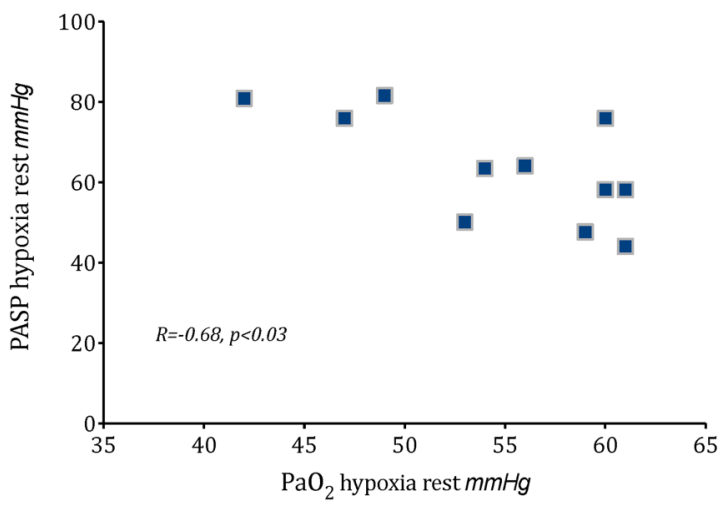

Figure 1 Correlation of pulmonary artery systolic pressure (PASP) and pressure of arterial oxygen $\left(\mathrm{PaO}_{2}\right)$ during resting hypoxia in 11 patients with pulmonary arterial hypertension.

heart parameters during all hypoxia stages $(p>0.6$, figure 3 ). In the control group, no significant changes in RV size and systolic function were also observed during all hypoxia stages, with the exception that TAPSE increased significantly $(p=0.03$ ). PASP increased from 24 (2) at baseline to $37 \quad(5) \mathrm{mm} \mathrm{Hg} \quad(\mathrm{p}<0.01)$ during hypoxia with exertion in the control group. RV parameters of individual patients at baseline, hypoxia at rest and hypoxia during exercise are shown in figure $3 . \mathrm{LV}$ ejection fraction did not differ between the PAH and control groups, at rest or during hypoxia stages $(\mathrm{p}>0.07)$.

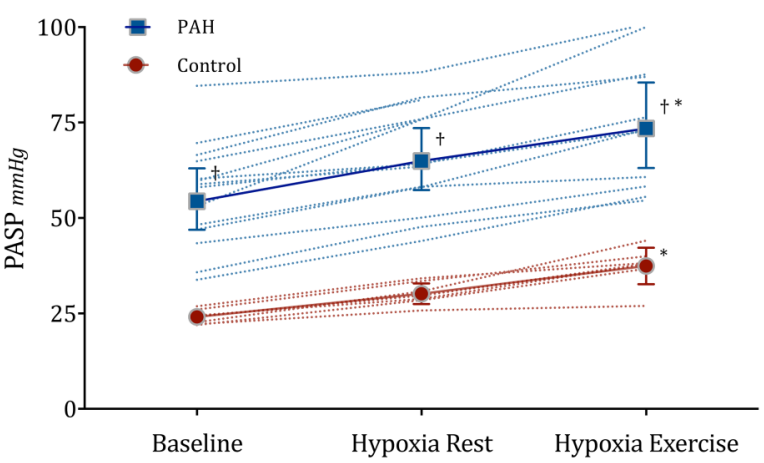

Figure 2 Mean pulmonary arterial systolic pressure (PASP) with $95 \% \mathrm{Cl}$ error bars and individual data points at rest breathing room air (baseline), during 20 min breathing 15.1\% $\mathrm{O}_{2}$ at rest (hypoxia rest) and following mild exercise (hypoxia exercise) in 14 patients with pulmonary arterial hypertension (PAH) and 7 healthy patients (control). ${ }^{*} \mathrm{p}<0.01$ ANOVA, $+\mathrm{Vs}$ Control $p<0.01$, unpaired $t$-test. $N=11$ PAH hypoxia during exercise. ANOVA, analysis of variance; $\mathrm{PAH}$, pulmonary arterial hypertension.

In the self-assessment of dyspnoea, the BORG scale increased only in patients with PAH and not the control patients during the hypoxia phases $(p<0.01)$. The patient who did not complete the exercise phase due to significant arterial desaturation reported the highest selfassessment that related to 'moderate' dyspnoea. 

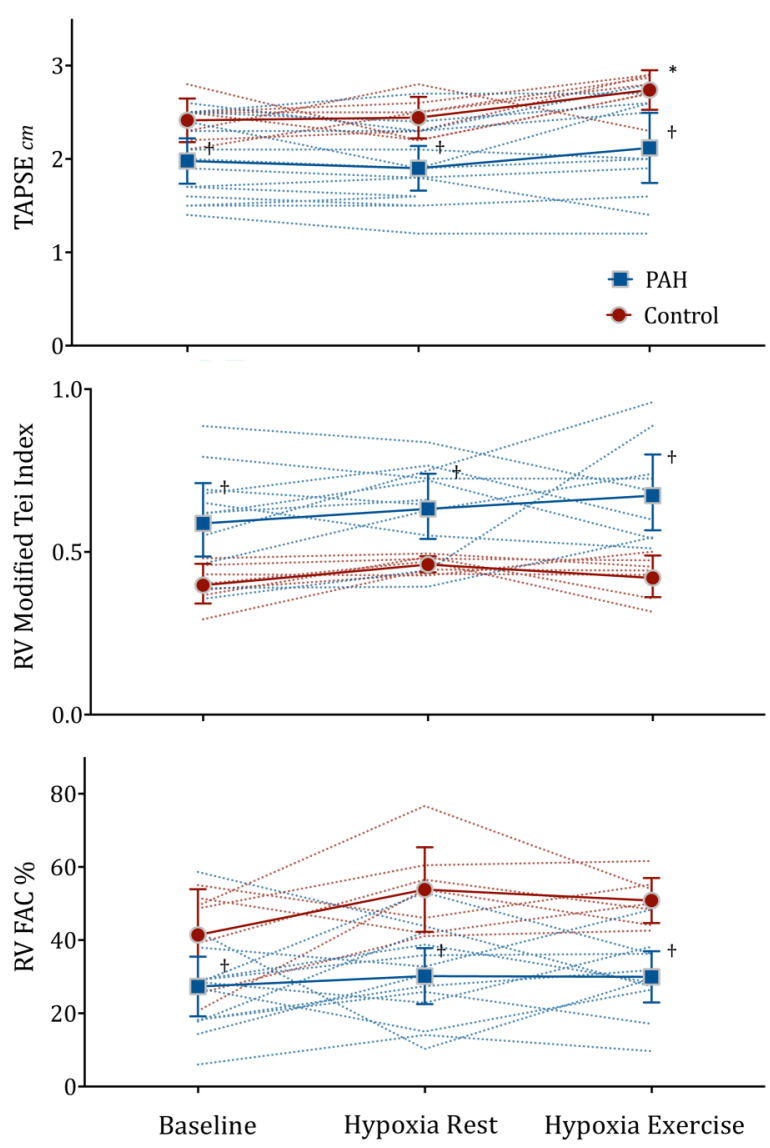

Figure 3 Mean with $95 \% \mathrm{Cl}$ error bars and individual data points of right ventricular function parameters at rest breathing room air (baseline), during 20 min breathing $15.1 \% \mathrm{O}_{2}$ at rest (hypoxia rest) and following mild exercise (hypoxia exercise) in 14 patients with pulmonary arterial hypertension (PAH) and 7 healthy patients (control). ${ }^{*} p<0.03$ ANOVA, $+V s$ Control $\mathrm{p}<0.04$, unpaired t-test. $\mathrm{N}=11 \mathrm{PAH}$ hypoxia during exercise. ANOVA, analysis of variance; FAC, fractional area change; $\mathrm{PAH}$, pulmonary arterial hypertension; RV, right ventricular;.

\section{DISCUSSION}

In patients with group $1 \mathrm{PAH}$ who were clinically stable on PAH-specific treatment, there was no evidence of progressive RV compromise during short-term simulated altitude at rest or under mild exertion. PASP increased in a predictable manner from baseline that was associated with arterial desaturation and a mild increase in the perception of breathlessness.

As far as we are aware, a study of right heart function using echocardiography during hypoxia in patients with pre-existing PAH has not been previously undertaken, at rest or during exercise. RV measures were significantly different in the patients with $\mathrm{PAH}$ as compared to controls during all stages; however, there were no changes in these parameters from baseline during hypoxia in either group. The exception was TAPSE, which increased only in the control group during exercise with hypoxia. A similar study performed on patients with chronic heart failure with a lower baseline PASP at sea level $(\approx 30 \mathrm{~mm} \mathrm{Hg})$ reported $\mathrm{a} \approx 4 \mathrm{~mm} \mathrm{Hg}$ rise in PASP following 1 hour at simulated $2400 \mathrm{~m}$ altitude at rest. ${ }^{26}$ The authors attributed this rise to a mildly elevated PVR that was also seen in the control group. In comparison to our study participants who have PAH with normal LV systolic function, their patients had predominantly impaired LV systolic dysfunction, with a large proportion of patients $(90 \%)$ on $\beta$-blockers. The latter would have limited a patient's ability to increase cardiac output due to chronotropic and inotropic blunting.

The patients with PAH in this study had moderately elevated PASP and the majority had evidence of RV dilation and RV dysfunction at baseline sea level. The observed group mean increase in PASP from baseline after mild exercise during hypoxia was $\approx 20 \mathrm{~mm} \mathrm{Hg}$. Despite this increase in PASP, we did not document any deterioration or change in measures of RV size or function on echocardiography, and no adverse events were reported despite a mild increase in the perception of breathlessness. This includes the single patient who did not complete the exercise stress due to significant desaturation. The postulated deleterious effects on RV function from hypoxic pulmonary vasoconstriction and/or $\mathrm{RV}$ ischaemia in patients with PAH were not apparent in this study. Hypoxia equivalent to commercial air travel $(2400 \mathrm{~m})$ may not have been sufficient to elicit detectable adverse effect on the RV. However, it should be noted that controls displayed augmentation of TAPSE during hypoxia with exertion, a phenomenon that was not observed in the PAH group. It has been demonstrated that an increase in TAPSE occurs during exercise in healthy controls. ${ }^{27}$ The lack of increase in TAPSE in the PAH group may indicate reduced RV reserve or possibly reflect early signs of hypoxic decompensation.

Our findings provide a basis for understanding observations in previous simulation and flight studies demonstrating that patients with $\mathrm{PAH}$ tolerate the low duration and low physical demands of air travel well ${ }^{6}{ }^{7}$ and that further direct invasive assessments of pulmonary and cardiac haemodynamics of longer or more significant hypoxic exposures can be safely considered. Our study protocol was consistent with the standard 'fit to fly' altitude simulation test for intolerance to hypoxia. ${ }^{28}$ This test duration is validated to achieve gas exchange equilibration in those with respiratory disease; however, it may not be sufficient to observe the development of any possible delayed adverse events of RV function in patients with PAH.

Pulmonary artery pressure was estimated using echocardiography rather than invasive gold standard right heart catheterisation assessment. Good agreement between the modalities has been demonstrated over a broad range of values at high altitudes up to $4600 \mathrm{~m} ;{ }^{29}$ however, generally, echocardiography is considered reproducible and accurate, but with moderate precision as compared to catheterisation techniques. ${ }^{30}$ Echocardiography was deemed appropriate for this primary pilot study given its noninvasive nature and the ability to simultaneously assess both RV function and PASP under hypoxic conditions. 
Patients with PAH desaturated to a greater extent from baseline during 'hypoxia at rest' than healthy patients and the pressure of arterial oxygen strongly correlated with PASP; three patients with a $\mathrm{PaO}_{2}$ that fell below $50 \mathrm{~mm} \mathrm{Hg}$ also recorded significantly elevated PASP and higher perceptions of breathlessness. Absolute minute ventilation and respiratory rate were higher in PAH in resting conditions compared to normal patients, possibly attributed to their reduced diffusing capacity that may have contributed to their higher perception of breathlessness.

It is acknowledged that our study population included mainly WHO FC II patients who were stabilised on PAH-specific therapies including ERA and/or PDE5-I. Thus, our study findings pertain strictly to this population which had well compensated disease. It is unknown whether a more prolonged period of hypoxia (beyond our protocol duration) will lead to deleterious effects on RV function, particularly considering the observed nonchanging TAPSE. Thus, our study findings do not contradict current recommended guidelines regarding the use of supplemental oxygen in flight. Similarly, further studies are needed to determine the effect of simulated altitude in patients with $\mathrm{PAH}$ with more advanced FC status.

\section{CONCLUSION}

Stable group 1 patients with $\mathrm{PAH}$ on vasodilator treatment did not experience any acute deterioration in $\mathrm{RV}$ function during simulated mild altitude, at rest or following mild exertion. PASP increased both at rest and during mild exercise that was associated with arterial desaturation. Despite a mild increase in symptoms, the level and duration of hypoxia in this study protocol was tolerated well.

Acknowledgements The authors thank Ms Sharon Quirk and Dr Tommy Chung of the Department of Cardiology, Concord Hospital, for their assistance with patient recruitment.

\section{Competing interests None declared.}

Ethics approval Sydney Local Area Health District, New South Wales, Australia, HREC.

Provenance and peer review Not commissioned; internally peer reviewed.

Open Access This is an Open Access article distributed in accordance with the Creative Commons Attribution Non Commercial (CC BY-NC 4.0) license, which permits others to distribute, remix, adapt, build upon this work noncommercially, and license their derivative works on different terms, provided the original work is properly cited and the use is non-commercial. See: http:// creativecommons.org/licenses/by-nc/4.0/

\section{REFERENCES}

1. Lau EM, Tamura Y, McGoon MD, et al. The 2015 ESC/ERS Guidelines for the diagnosis and treatment of pulmonary hypertension: a practical chronicle of progress. Eur Respir $J$ 2015;46:879-82.

2. Galie N, Humbert M, Vachiery JL, et al. 2015 ESC/ERS Guidelines for the diagnosis and treatment of pulmonary hypertension: the Joint Task Force for the Diagnosis and Treatment of Pulmonary Hypertension of the European Society of Cardiology (ESC) and the
European Respiratory Society (ERS) Endorsed by: Association for European Paediatric and Congenital Cardiology (AEPC), International Society for Heart and Lung Transplantation (ISHLT) Eur Heart J 2016;37:67-119.

3. Ahmedzai S, Balfour-Lynn IM, Bewick T, et al. Managing passengers with stable respiratory disease planning air travel: British Thoracic Society recommendations. Thorax 2011;66 (Suppl 1):i1-30.

4. Code of Federal Regulations. 14, part 25841. Washington: US Goverment Printing Office, 1986.

5. Kelly PT, Seccombe LM, Rogers PG, et al. Directly measured cabin pressure conditions during Boeing 747-400 commercial aircraft flights. Respirology 2007;12:511-15.

6. Roubinian N, Elliott CG, Barnett CF, et al. Effects of commercial air travel on patients with pulmonary hypertension air travel and pulmonary hypertension. Chest 2012;142: 885-92.

7. Burns RM, Peacock AJ, Johnson MK, et al. Hypoxaemia in patients with pulmonary arterial hypertension during simulated air travel. Respir Med 2013;107:298-304.

8. Vogel JA, Harris CW. Cardiopulmonary responses of resting man during early exposure to high altitude. J Appl Physiol 1967;22:1124-8.

9. Talbot NP, Balanos GM, Dorrington KL, et al. Two temporal components within the human pulmonary vascular response to approximately $2 \mathrm{~h}$ of isocapnic hypoxia. J Appl Physiol 2005;98:1125-39.

10. Swenson ER. Hypoxic pulmonary vasoconstriction. High Alt Med Biol 2013;14:101-10.

11. Groves BM, Reeves JT, Sutton JR, et al. Operation Everest II: elevated high-altitude pulmonary resistance unresponsive to oxygen J Appl Physiol 1987;63:521-30.

12. Huez S, Retailleau K, Unger P, et al. Right and left ventricular adaptation to hypoxia: a tissue Doppler imaging study. Am J Physiol Heart Circ Physiol 2005;289:H1391-8.

13. McLaughlin VV, Archer SL, Badesch DB, et al. ACCF/AHA 2009 expert consensus document on pulmonary hypertension a report of the American College of Cardiology Foundation Task Force on Expert Consensus Documents and the American Heart Association developed in collaboration with the American College of Chest Physicians; American Thoracic Society, Inc.; and the Pulmonary Hypertension Association. J Am Coll Cardiol 2009;53:1573-619.

14. Miller MR, Hankinson J, Brusasco V, et al. Standardisation of spirometry. Eur Respir J 2005;26:319-38.

15. Macintyre N, Crapo RO, Viegi G, et al. Standardisation of the single-breath determination of carbon monoxide uptake in the lung. Eur Respir J 2005;26:720-35.

16. Quanjer PH, Stanojevic S, Cole TJ, et al. Multi-ethnic reference values for spirometry for the 3-95-yr age range: the global lung function 2012 equations. Eur Respir J 2012;40: 1324-43.

17. Quanjer PH. Standardized lung function testing. Report working party. Bull Eur Physiopathol Respir 1983;19(Suppl 5):1-95.

18. Lang RM, Badano LP, Mor-Avi V, et al. Recommendations for cardiac chamber quantification by echocardiography in adults: an update from the American Society of Echocardiography and the European Association of Cardiovascular Imaging. Eur Heart $J$ Cardiovasc Imaging 2015;16:233-70.

19. Wright LM, Dwyer N, Celermajer D, et al. Follow-up of pulmonary hypertension with echocardiography. JACC Cardiovasc Imaging 2016;9:733-46.

20. Forfia PR, Fisher MR, Mathai SC, et al. Tricuspid annular displacement predicts survival in pulmonary hypertension. Am J Respir Crit Care Med 2006;174:1034-41.

21. Harada $\mathrm{K}$, Tamura $\mathrm{M}$, Toyono $\mathrm{M}$, et al. Comparison of the right ventricular Tei index by tissue Doppler imaging to that obtained by pulsed Doppler in children without heart disease. Am J Cardiol 2002;90:566-9.

22. Rudski LG, Lai WW, Afilalo J, et al. Guidelines for the echocardiographic assessment of the right heart in adults: a report from the American Society of Echocardiography endorsed by the European Association of Echocardiography, a registered branch of the European Society of Cardiology, and the Canadian Society of Echocardiography. J Am Soc Echocardiogr 2010;23:685-713; quiz 86-8.

23. Chow V, Ng ACC, Seccombe L, et al. Impaired 6-min walk test, heart rate recovery and cardiac function post pulmonary embolism in long-term survivors. Respir Med 2014;108:1556-65. 
24. Jette M, Sidney K, Blumchen G. Metabolic equivalents (METS) in exercise testing, exercise prescription, and evaluation of functional capacity. Clin Cardiol 1990;13:555-65.

25. Borg GA. Psychophysical bases of perceived exertion. Med Sci Sports Exerc 1982;14:377-81.

26. Hobkirk JP, Damy T, Walters M, et al. Effects of reducing inspired oxygen concentration for one hour in patients with chronic heart failure: implications for air travel. Eur J Heart Fail 2013;15:505-10.

27. Chia EM, Lau EM, Xuan W, et al. Exercise testing can unmask right ventricular dysfunction in systemic sclerosis patients with normal resting pulmonary artery pressure. Int J Cardiol 2016;204:179-86.
28. Gong $\mathrm{H}$ Jr, Tashkin DP, Lee EY, et al. Hypoxia-altitude simulation test. Evaluation of patients with chronic airway obstruction. Am Rev Respir Dis 1984;130:980-6.

29. Allemann Y, Sartori C, Lepori M, et al. Echocardiographic and invasive measurements of pulmonary artery pressure correlate closely at high altitude. Am J Physiol Heart Circ Physiol 2000;279: H2013-16.

30. D'Alto M, Romeo E, Argiento P, et al. Accuracy and precision of echocardiography versus right heart catheterization for the assessment of pulmonary hypertension. Int J Cardiol 2013;168:4058-62. 